\title{
Estrategias de desarrollo de la competencia transversal sobre Responsabilidad Medioambiental en alumnos de Grado
}

\author{
$M^{a}$ Ángeles Alcaide ${ }^{a}$ y Elena De la Poza ${ }^{b}$ \\ a,b Centro de Ingeniería Economía, Universitat Politècnica de València, ${ }^{a}$ manalgon@upv.es, \\ belpopla@esp.upv.es.
}

\begin{abstract}
Nowadays, we are almost all aware that climate change is taking place on the planet, but not all of us are environmentally responsible enough yet. Our objective is to evaluate this environmental commitment in first-year undergraduate students, given that the ethical, environmental and professional responsibility is the control point of the subject, in order to establish strategies for the development of environmental responsibility. For this, it is observed if the students are able to make a decision respectful with the environment that will affect the project that must be done throughout the course in the subject using the Project based learning methodology (PBL), and will be contrasted with the opinions collected at the beginning of the course about their habits and environmental awareness.
\end{abstract}

Keywords: transversal competences, environmental responsibility, environmental commitment, project based learning, PBL, sustainable habits.

\begin{abstract}
Resumen
Hoy en día prácticamente todos somos conscientes de que se está produciendo un cambio climático en el planeta, sin embargo todavía no todos somos lo suficientemente responsables medioambientalmente. Nuestro objetivo es evaluar ese compromiso medioambiental en alumnos de primer curso de Grado, dado que la competencia responsabilidad ética, medioambiental y profesional es punto de control de la asignatura, con el fin de establecer estrategias de desarrollo de la responsabilidad medioambiental. Para ello, se observa si los alumnos son capaces de tomar una decisión respetuosa con el medio ambiente que afectará al proyecto que deben realizar a lo largo del curso en la asignatura empleando la metodología Project based learning (PBL), y se contrastará con las opiniones recogidas al inicio de curso sobre sus habitos y conciencia medioambiental.
\end{abstract}

Palabras clave: competencias transversales, responsabilidad ambiental, compromiso medioambiental, project based learning, PBL, hábitos sostenibles.

\section{Introducción}

La competencia Responsabilidad Ética, Medioambiental y Profesional, tal como se define en el proyecto de competencias transversales de la UPV (UPV, 2019) es "el conjunto de 
conocimientos, habilidades, destrezas y actitudes, útiles para interactuar con el entorno, de forma ética, responsable y sostenible, en orden a evitar o disminuir los efectos negativos producidos por las prácticas inadecuadas que ocasiona la actividad humana y para promover los beneficios que pueda generar la actividad profesional en el ámbito medioambiental, teniendo en cuenta sus implicaciones económicas y sociales." Además, este mismo proyecto distingue los tres tipos de responsabilidades: (i) la ética que relaciona la acción humana con su valor moral; (ii) la medioambiental, la cual valora el impacto ecológico causado por otro individuo o grupo; y (iii) la profesional que vincula nuestra propia responsabilidad moral para evitar cualquier falta voluntaria y disminuir aquellas faltas involutarias por debilidad humana, flaqueza propia o negligencia ajena.

Esta competencia se trabaja en la asignatura de Empresa (primer curso) del Grado de Ingeniería Aeroespacial impartido en la Escuela Técnica Superior de Ingeniería del Diseño de la Universitat Politècnica de València en dos dimensiones: por una parte, la responsabilidad ética y profesional y, por otra parte, la responsabilidad medioambiental. En esta comunicación nos centramos únicamente en esta segunda parte: la Responsabilidad Medioambiental.

De esta manera, entendemos la responsabilidad medioambiental como la manera de evaluar nuestro compromiso con el planeta. Este compromiso es de todos: personas, empresas, organizaciones y cualquier otro agente.

Así pues, la responsabilidad medioambiental es una competencia transversal que debe estar presente en todo momento de la vida de las personas, y a lo largo de todas las fases de la Educación Superior, pero especialmente en los primeros años, ya que cuanto antes se cree una conciencia sostenible, antes se actuará paliando los efectos negativos en nuestro entorno. Sin embargo, hay diferentes medios para desarrollar y evaluar esta competencia. Varios trabajos (Lans et al, 2014; Lozano et al, 2019) han utilizado cuestionarios sobre los estudiantes para evaluarla. Aunque con enfoques y resultados diversos. El primer trabajo utilizó los cuestionarios para desarrollar un conjunto de competencias claras y distintas para valorar la responsabilidad medioambiental y el espíritu sostenible de los estudiantes. El segundo también utilizó los cuestionarios para investigar el desarrollo de competencias sostenibles, pero en este caso distinguiendo entre la 3 dimensiones de la sostenibilidad: ambiental, social y económica. En este caso, los resultados mostraron una relación entre la sostenibilidad y las competencias, y entre las competenicas y sus aplicaciones pedagógicas, además ayudaron a desarrollar mejor dichas competencias. Estos resultados tienen coherencia con los de Lambrechts et al (2013), quienes también concluyeron que se pueden descubrir muchas competencias para la responsabiliad medioambiental o sostenible dentro de los programas de educación superior, aunque ellos apuntaron que sólo de forma implícita y fragmentada, por lo que no cubren todos los campos necesarios de conocimiento, habilidades y actitudes.

Otros trabajos (Sidiropoulos, 2014) describen prácticas educativas concretas para incorporar la responsabilidad medioambiental en las aulas de educación superior. Estas prácticas van desde la integración incremental (como la discusión en clase sobre temas del currículo) hasta 
cursos específicos (como seminarios introductorios de sostenibilidad para una integración más profunda de la sostenibilidad en el currículo y en la evaluación del curso).

Observamos que no hay un método o criterio comúnmente aceptado en la literatura, sino que se distinguen varias alternativas. Es por ello, que para evaluar esta competencia, en primer lugar, proponemos que el docente realice un análisis inicial del estado de conocimiento y comprensión de dicha competencia por parte del alumno. De igual forma que el docente analiza el nivel de conocimiento con que el alumno accede a la asignatura, y en base a las competencias específicas de la misma, define si es necesario que el alumno acceda a la asignatura tras haber superado unos prerrequisitos; lo mismo ocurre en el caso de las competencias transversales, para las cuales el docente evalua el punto de partida del alumno, y en función de éste, determina las estrategias de aprendizaje más adecuadas.

En esta contribución nos centramos en el análisis de la responsabilidad medioambiental del alumnado al inicio de sus estudios universitarios como forma de determinar su nivel de compromiso y conocimiento con la responsabilidad medioambiental y así definir las mejores estrategias de desarrollo y aprendizaje de esta competencia transversal.

Para analizar el punto de partida de responsabilidad medioambiental del alumno, se ha diseñado una encuesta que los alumnos contestan el primer día de clase de la asignatura de Empresa, y a través de la que se miden sus hábitos y conciencia medioambiental.

En segundo lugar, y en base a los resultados obtenidos en la encuesta, se propone al alumno para el desarrollo de la competencia transversal en materia medioambiental un proyecto innovador basado en la creación de un modelo de negocio que promueva la sostenibilidad y se integre en el ámbito de sus estudios (Grado en Ingeniería Aeroespacial), bien a través de la mitigación de los efectos negativos del cambio climático o mediante el desarrollo de los aspectos positivos.

Esta estrategia de aprendizaje promueve no solo el desarrollo de la competencia transversal $\mathrm{N}^{\circ} 07$ sino también la competencia $\mathrm{N}^{\circ} 04$ sobre innovación, creatividad y emprendimiento, ya que el alumno desarrolla una idea empresarial a partir de los conocimientos adquiridos durante el curso. En su defecto, los alumnos que no desean desarrollar un modelo de negocio para la sostenibilidad, eligen un proyecto tradicional, basado en el análisis del plan de negocio de una empresa consolidada pertenciente al sector aeronáutico previamente determinada por los docentes de la asignatura, y como forma de desarrollo de la competencia transversal en su componente medioambiental, el alumno analiza los informes de responsabilidad social corporativa (RSC) de la empresa a lo largo del tiempo. De esta manera, trataremos de analizar si los alumnos que afirman tener interés por la sostenibilidad, realmente cuando se les da la oportunidad, lo hacen, y son capaces de generar modelos de negocio que fomenten la sostenibilidad.

Así, la metodología de aprendizaje de los alumnos está basada en el Project Based Learning (PBL) o Aprendizaje Basado en Proyectos, la cual se puede definir como una metodología docente centrada en el estudiante, donde se trabaja en grupos de forma coordinada, ejecutando proyectos realistas y prácticos, desarrollando un amplio marco de habilidades y aplicando los conocimientos adquiridos de forma multidisciplinar (ICE, 2019). Como 
ejemplo, en el trabajo de Orozco-Messana y De la Poza (2018) se aborda la conceptualización, diseño e implementación de la metodología PBL aplicada a un taller sobre sostenibilidad.

\section{Objetivos}

El objetivo de este trabajo es valorar la responsabilidad medioambiental de los alumnos de primer curso de grado en Ing. Aeroespacial para determinar su grado de compromiso con dicha competencia medioambiental, y con la finalidad de desarrollar estrategias de aprendizaje para el desarrollo de la misma cuidando el componente motivador por la asignatura basado en la metodología PBL promoviendo el "learning by doing".

De esta forma, este trabajo trata de aportar evidencias de cómo la Educación Superior contribuye al desarrollo integral del alumnado, no solo valorando el contenido curricular de las materias, sino también otras competencias que van más allá de dichos contenidos, y que capacitan a los alumnos para su integración en el mundo profesional y en la sociedad actual.

\section{Desarrollo de la innovación}

Para desarrollar nuestro objetivo: analizar el conocimiento de los alumnos en la competencia de responsabilidad ambiental al inicio de curso con el fin de desarrollar estrategias de enseñanza-aprendizaje que mejor se adapten a su perfil, nos basamos en dos herramientas. En primer lugar, una encuesta realizada al inicio del curso compuesta por 8 preguntas sobre sus hábitos y conciencia medioambiental. Y en segundo lugar, en observar la decisión de los alumnos a la hora de seleccionar de manera voluntaria el proyecto basado en la metodología PBL y desarrollado en la asignatura a lo largo del curso, ya que pueden decidir hacer un proyecto innovador sobre un modelo de negocio que sea generador de sostenibilidad, o bien un proyecto tradicional basado en un plan de empresa.

Así, hay que tener en cuenta que este proyecto se realiza en grupos de 5 alumnos, por lo que la decisión al seleccionar el tipo de proyecto es conjunta. De esta manera, la hipótesis que se quiere comprobar es que los alumnos que inician el curso con conocimientos previos en materia medioambiental y conciencia sobre la responsabilidad medioambiental de sus acciones, cuando se les da la oportunidad, participan activamente en el desarrollo de un plan de empresa que mitigue los efectos del cambio climático. En cambio, es esperable que los alumnos sin conciencia medioambiental opten por un aprendizaje guiado a través del análisis del plan de empresa y también desarrollen la competencia $\mathrm{N}^{\circ} 07$ a través del análisis de los informes RSC de una firma consolidada .

En consecuencia nuestra hipótesis de investigación (H1) es: el alumno con conciencia ambiental participa activamente en el desarrollo de un modelo de negocio para la sostenibilidad en el ámbito del sector aeronáutico en la asignatura de Empresa, ampliando sus conocimientos de forma activa y autónoma.

La encuesta realizada a los alumnos está estructurada de la siguiente manera: 


\section{Tabla 1. Modelo de cuestionario}

1. Sexo:
a. $\mathrm{M}$
b. $\mathrm{F}$

2. Edad:

3. ¿Crees que se está produciendo un cambio climático en el planeta?
a. $\mathrm{Si}$
b. No

4. ¿Has estudiado o sabes algo sobre medioambiente?
a. Sí
b. Sólo tengo conocimientos básicos
c. No

5. Si contestaste que sí a la 4, ¿Dónde lo aprendiste?
a. Educación Primaria.
b. Educación Secundaria.
c. Conferencias o cursos específicos.
d. Noticias.
e. Búsqueda por Internet.

6. ¿Reciclas los residuos?
a. Sí
b. No

7. Si contestaste que sí a las 6 , ¿Por qué reciclas?
a. Ayudar al planeta y al medioambiente.
b. Por tradición, siempre lo he hecho.
c. Para ayudar a las generaciones futuras.
d. Otros.

8. Si contestaste que no a la 6 , ¿Por qué no reciclas?
a. Por tradición, en mi casa nunca se ha hecho.
b. No lo hago, pero creo que en un futuro cercano lo haré.
c. Por falta de medios.

Las dos primeras preguntas clasifican demográficamente a la muestra según su sexo y edad. Las preguntas 3, 4 y 6 son de respuesta dicotómica, siendo 1 si la respuesta es afirmativa y 0 si es negativa. Las preguntas 5,7 y 8 son preguntas de respuesta abierta, y que posteriormente han sido codificadas en preguntas cerradas categóricas de opción múltiple, es decir, que las respuestas no son excluyentes entre sí, resultando la pregunta 5 con 5 respuestas, la pregunta 7 con 4 respuestas, y la 8 con 3 respuestas.

Las encuestas o cuestionarios han sido empleados en la evaluación de las competencias en multitud de trabajos (Martí \& Yepes, 2015; Solanes et al, 2008). 
El análisis se realiza sobre una muestra constituida por los alumnos matriculados en el grupo de alto rendimiento académido (grupo ARA) de primer año del Grado en Ing. Aeroespacial de la UPV (curso académico 2018/2019). EL alumno accede al grupo ARA por la nota de su expediente académico. De hecho, la nota media de los alumnos que logran una plaza en un grupo ARA en la UPV $(11,2)$ es superior a la nota media del resto de grupos $(9,2)$. A su vez, la nota media de acceso al grupo ARA del Grado en Ing. Aeroespacial supera la nota media de acceso a los grupos ARA de la UPV.

La muestra se compone de un total de 56 alumnos, 13 mujeres y 43 hombres. Todos ellos entre 17 y 18 años, y en su mayor parte procedentes de la ciudad de Valencia (España). Sin embargo uno de ellos no contestó ninguna de las preguntas del cuestionario, por lo que lo excluímos del análisis, reduciendo la muestra a 55 alumnos.

\section{Resultados}

A continuación se muestran los resultados obtenidos de la encuesta realizada al inicio de curso de forma gráfica.

La tabla 2 nos indica que el $98,18 \%$ de los alumnos es conciente de que se está produciendo un cambio climático en el planeta. La tabla 3 muestra como el 63,6\% de los alumnos han estudiado o tienen conocimientos del medio ambiente; sin embargo, el 23,6\% afirma que solo tiene conocimientos básicos, y el restante $13 \%$ confirma que no tiene ningún conocimiento o estudios sobre el medio ambiente. De los que afirman que tienen algún conocimiento sobre medio ambiente (un $87,2 \%$ de los alumnos), el 34,5\% afirma que los ha adquirido en educación primaria, el $25,45 \%$ en educación secundaria, el 14,5\% a través de la búsqueda por si mismo en Internet, el $12,7 \%$ a través de conferencias o cursos específicos, y el mismo porcentaje para los que afirman que han adquirido dichos conocimientos a través de noticias en los medios de comunicación (figura 1). Es decir, el 40\% de los alumnos ha desarrollado sus conocimientos sobre medioambiente de forma autónoma, sin formar parte de una estrategia de aprendizaje vinculada al sistema educativo.

Tabla 2. Análisis de la pregunta 3 del cuestionario

¿Crees que se está produciendo un

cambio climático en el planeta?

\begin{tabular}{cc}
\hline Sí & $98,18 \%$ \\
No & $1,81 \%$ \\
\hline
\end{tabular}

Tabla 3. Análisis de la pregunta 4 del cuestionario

¿Tienes conocimientos sobre medioambiente?

(c) B BY-NC-ND 2019, Universitat Politècnica de València 


\begin{tabular}{cc}
\hline Sí & $63,60 \%$ \\
Básico & $23,63 \%$ \\
No & $13 \%$ \\
\hline
\end{tabular}

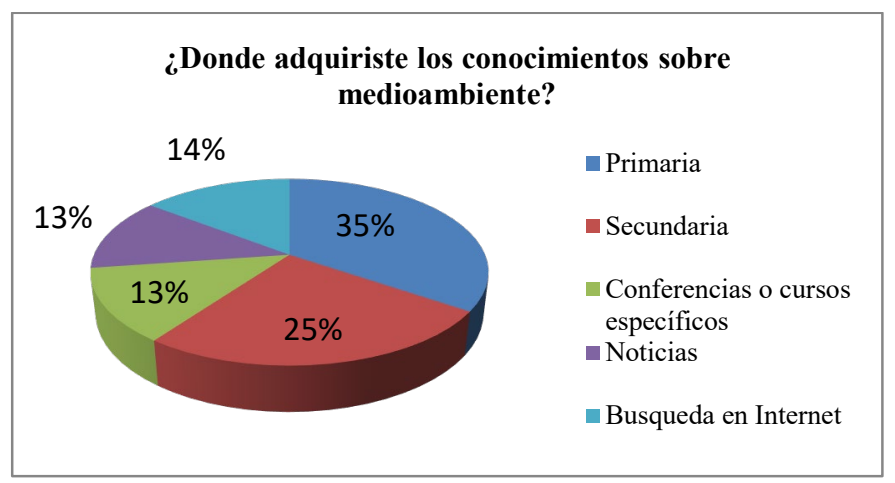

Fig. 1 Análisis de la pregunta 5 del cuestionario

Tabla 4. Análisis de la pregunta 6 del cuestionario

\begin{tabular}{lr}
\hline \multicolumn{2}{c}{ ¿Reciclas los residuos? } \\
\hline Sí & $80 \%$ \\
No & $20 \%$ \\
\hline
\end{tabular}

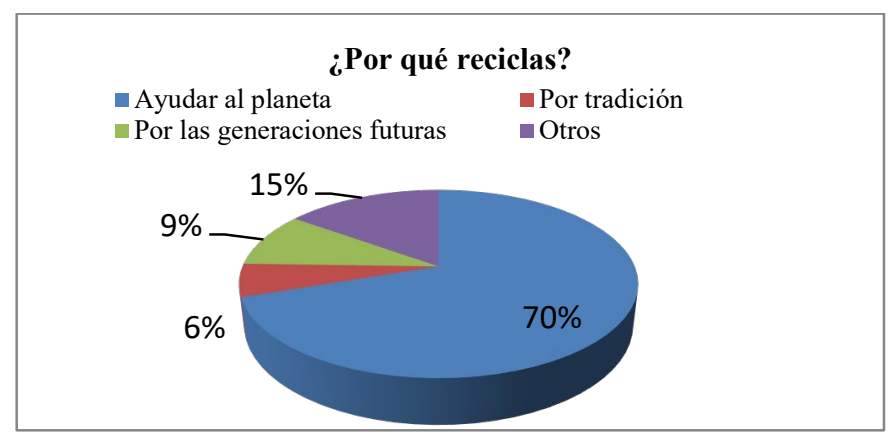

Fig. 2 Análisis de la pregunta 7 del cuestionario

Tabla 5. Análisis de la pregunta 8 del cuestionario ¿Por qué no reciclas? 


\begin{tabular}{cc}
\hline No tengo medios & $14,30 \%$ \\
Plan futuro & $35,70 \%$ \\
Por tradición & $50 \%$
\end{tabular}

A la pregunta sobre si reciclan los residuos (tabla 4), el 80\% de los alumnos confirma que sí lo hace, mientras que el $20 \%$ restante no lo hace. De los 44 alumnos que confirman reciclar los residuos, el 69,8\% afirma que lo hace para ayudar al planeta y a nuestro medioambiente, el 9,4\% para contribuir a las generaciones futuras, el 5,6\% por costumbre o tradición porque es algo que lo ha hecho desde siempre, y el restante $15,1 \%$ por otros motivos (figura 2 ). Por último, en la tabla 5 se observa que, de los 11 alumnos que contestaron que no reciclan los residuos, el 50\% indican que no lo hacen por tradición, porque en sus familias nunca se ha hecho, el 36\% revela que no reciclan pero que sí que lo consideran hacer en un futuro, y el $14 \%$ apuntan que no reciclan porque no tienen medios en su entorno, por ejemplo no tienen contenedores para clasificar los diferentes tipos de residuos.

En segundo lugar, se observa que el 50,9\% de los alumnos decidió realizar un proyecto innovador que incluyera la responsabilidad medioambiental en la asignatura a través de la metodología PBL, mientras que el $49,1 \%$ restante decidió realizar un proyecto sobre un modelo guiado de plan de empresa incluyendo la competencia medioambiental a través del análisis de los informes de RSC de la compañía analizada.

Por último, realizamos un análisis mediante tabulación cruzada entre los alumnos que sí deciden realizar un proyecto innovador y las preguntas dicotómicas del cuestionario, que son la número 3 , la 4 y la 6 , sobre si creen que se está produciendo un cambio climático, si tienen conocimientos sobre el medio ambiente, y si reciclan, respectivamente. Los resultados (tabla 6) del análisis indican que:

Tabla 6. Resultados del análisis por tabulación cruzada en valores absolutos y relativos

\begin{tabular}{|c|c|c|c|}
\hline \multicolumn{2}{|c|}{} & $\begin{array}{c}\text { PBL innovador que } \\
\text { promueve la sostenibilidad }\end{array}$ & $\begin{array}{c}\text { PBL sobre plan } \\
\text { empresa tradicional }\end{array}$ \\
\hline $\begin{array}{c}\text { ¿Crees que se está } \\
\text { produciendo un } \\
\text { cambio climático? }\end{array}$ & Sí & $28 / 51,85 \%$ & $26 / 48,14 \%$ \\
\cline { 2 - 4 } & No & $0 / 0 \%$ & $1 / 100 \%$ \\
\hline $\begin{array}{c}\text { ¿Tienes } \\
\text { menocimientos sobre }\end{array}$ & Sí & $23 / 47,97 \%$ & $25 / 52,08 \%$ \\
\cline { 2 - 4 } & No & $5 / 71,42 \%$ & $21 / 47,72 \%$ \\
\hline \multirow{2}{*}{$\begin{array}{c}\text { ¿Reciclas los } \\
\text { residuos? }\end{array}$} & Sí & $23 / 52,27 \%$ & $6 / 54,54 \%$ \\
\cline { 2 - 4 } & No & $5 / 45,45 \%$ & $28,57 \%$ \\
\hline
\end{tabular}

Fuente: Elaboración propia.

- De los alumnos que sí que creen que se está produciendo un cambio climático, el $51,85 \%$ elegió realizar un proyecto innovador para incorporar la responsabilidad 
ambiental en el mismo. Sin embargo, sólo 1 alumno contestó que no cree que se esté produciendo un cambio climático, el cual desarrolló el PBL sobre un plan de empresa tradicional.

- De los alumnos que sí que tienen conocimientos sobre el medio ambiente, el $47,97 \% \%$ elegió realizar un proyecto innovador para incorporar la responsabilidad ambiental. No obstante, de los alumnos que no tenían tales conocimientos, un $71,42 \%$ también decidió realizar este tipo de proyecto.

- De los alumnos que sí que reciclan, el 52,27\% elegió realizar un proyecto innovador para incorporar la responsabilidad ambiental. En cambio, de los que no tienen costumbre de reciclar los residuos, un 45,45\% también realizó el proyecto innovador.

\section{Conclusiones}

El 98\% de los alumnos del grupo ARA de la asignatura Empresa impartida en el Grado de Ingeniería Aeroespacial en el curso 2018/2019 cree que se está produciendo un cambio climatico, el $87 \%$ tiene algún conocimiento sobre medioambiente, y el $80 \%$ afirma que recicla, por lo que se observa un alto porcentaje de alumnos con conocimientos sobre medioambiente y también con una consciencia creada sobre el cambio climático y la importancia de contribuir para paliar los efectos negativos sobre el mismo.

Cuando se les da la oportunidad, el 50,9\% de alumnos adoptan una actitud proactiva y han propuesto modelos de negocio para la sostenibilidad. Sin embargo, hay un resultado equilibrado entre los que realizan este proyecto innovador y los que prefieren realizar un modelo guíado de plan de empresa incluyendo la competencia medioambiental a través del análisis de los informes de RSC de la compañía analizada.

Entre los que manifiestan una mayor concienciación y sensibilidad por el medio ambiente, ya que sí que creen que se esté produciendo un cambio climático, tienen conocimientos sobre el mismo, y reciclan los residuos, se observan unos resultados muy equitativos entre los que adoptan la actitud proactiva a través de su proyecto a lo largo del curso y los que no lo hace. Sin embargo, entre los que no manifiestan tener conocimientos, se aprecia una inquietud por adquirirlos, debido a que el $71,42 \%$ también decidió realizar el proyecto innovador generador de sostenibilidad.

Concluimos que un alto porcentaje de alumnos accede a la Educación Superior Universitaria manifestando competencias sufientes sobre responsabilidad medioambiental, sin embargo, cuando se les da la oportunidad de adoptar una actitud activa en el aula que desarrolle y potencie dicha competencia, obtenemos resultados no del todo satisfactorios, entendiendo como éxito, la iniciativa emprendedora en el desarrollo de modelos para la sostenibilidad. No obstante, se aprecia una actitud activa entre los que no tienen conocimientos sobre medio ambiente y los que no reciclan los residuos. 


\section{Referencias}

INSTITUTO DE CIENCIAS DE LA EDUCACIÓN consultado a través de UNIVERSITAT POLITÈCNICA DE CATALUNYA (2019). ¿Qué es PBL?. $<$ https://www.upc.edu/rima/es/grupos/gipbl-grupo-de-interes-en-ensenanza-basada-en-proyectosproblemas/bfque-es-pbl $>$ [Consutado: 10 de febrero de 2019]

LAMBRECHTS, W., MULÀ, I., CEULEMANS, K., MOLDEREZ, I., GAEREMYNCK, V. (2013). "The integration of competences for sustainable development in higher education: An analysis of bachelor programs in management". Journal of Cleaner Production, 48, 65-73.

LANS, T., BLOK, V., WESSELINK, R. (2014). "Learning apart and together: towards an integrated competence framework for sustainable entrepreneurship in higher education".Journal of Cleaner Production, 62, 37-47. DOI:10.1016/j.jclepro.2013.03.036.

LOZANO, R., BARREIRO-GEN, M., LOZANO, F.J., SAMMALISTO, K. (2019). “Teaching Sustainability in European Higher Education Institutions: Assessing the Connections between Competences and Pedagogical Approaches". Sustainability, 11, 1602.

MARTÍ, J.V., y YEPES, V. (2015). "Valoración de la competencia transversal Pensamiento Crítico por los alumnos de GIOP" en XIV Jornadas de Redes de Investigación en Docencia Universitaria. Investigación, Innovación y Enseñanza Universitaria: Enfoques Pluridisciplinares, Universidad de Alicante.

OROZCO-MESSANA, J. y DE LA POZA, E. (2018). "The ISA Lab workshop: a Project based learning iniciative" en $4^{\text {th }}$ International Conference on Higher Education Advances (HEAD), Universitat Politècnica de València.

SIDIROPOULOS, E. (2014). "Education for sustainability in business education programs: a question of value". Journal of Cleaner Production, 85, 472-487.DOI:10.1016/j.jclepro.2013.10.040.

SOLANES, A., NÚÑEZ, R., y RODRÍGUEZ, J. (2008). "Elaboración de un cuestionario para la evaluación de competencias genéricas en estudiantes universitarios" en Apuntes de Psicología, 26, 1, $35-49$.

UNIVERSITAT POLITÈCNICA DE VALÉNCIA (2019). La responsabilidad ética, medioambiental y profesional como competencia transversal. $<$ http://www.upv.es/contenidos/COMPTRAN/info/954882normalc.html $>$ [Consutado: 8 de febrero de 2019] 